\title{
Administration of anesthetic drugs according to pharmacological principles: are we heading in the right direction?
}

\author{
Hugo E. M. Vereecke ${ }^{1,2}$
}

Received: 2 August 2019 / Accepted: 2 August 2019 / Published online: 16 August 2019

(c) Springer Nature B.V. 2019

In this edition of JCMCI, two related papers have been published respectively exploring the feasibility [1] and the acceptability [2] of a new algorithm for the optimization of intravenous propofol titration in combination with remifentanil and fentanyl. Based on the demographic data of the patient and the timing and magnitude of the doses administered, the time course of the effect-site concentration has been calculated for each drug administered. These effect-site concentrations than serve as input for response surface druginteraction models that are elegant mathematical solutions to quantify the nature of drug interactions between drugs. Many clinical effects evoked by combinations of propofol and opioids have been studied this way, such as the probability of responsiveness to a verbal, tactile or noxious stimulation; or endpoints related to respiratory function; or residual analgesic effect [3-5]. By combining the predictions of models, the authors developed an advisory system for clinicians, that proposes dose adjustments in 20-min intervals to target an optimization of the recovery conditions for the patient. The proof of concept of this new algorithm is provided by demonstrating a theoretical advantage, being a prolongation of the time between end of surgery and the first need for additional analgesia, without causing excessive delays in respiratory function recovery. The authors confirm that the algorithm could be tweaked successfully to reach these predefined goals. In a second paper, the acceptability of the proposed dose adjustments by the new algorithm was tested in major spine surgery cases in a population of adolescent patients [2]. Thams et al. found a very high acceptance rate, suggesting that the algorithm proposes dose adjustments that are within normal clinical ranges, as perceived

Hugo E. M. Vereecke

Hugo.Vereecke@azsintjan.be; H.E.M.Vereecke@UMCG.Nl

1 University Medical Center Groningen and University of Groningen, Groningen, The Netherlands

2 Department of Anesthesia and Reanimation, AZ Sint-Jan Brugge-Oostende AV, Brugge, Belgium by experienced anesthesiologists. This primary endpoint is not sufficiently objective to confirm adequate performance of the algorithm, but it shows the willingness of clinicians to guide drug titration by means of theoretical models. The authors confirm that several methodological issues may have contributed to this positive result, and therefore the conclusion needs to be put in perspective.

The methodology used for developing the algorithm may also include a degree of positive bias, especially if the algorithm will be dependent on human interaction when used in "real life" clinical practice. First, a computer simulation uses input of drug administration rates with a $100 \%$ accuracy for dosing and timing, which will lead to the lowest possible prediction-errors in the estimation of the corresponding effect-site concentration and subsequently the most accurate prediction of the interaction effect from combined drugs. However, in clinical practice, the input of data might be diminished by human performance, as humans are notoriously less consistent in task performance due to fatigue, distraction or lack of concentration compared to computers. A higher prediction error may also originate from the $20 \mathrm{~min}$ interval between dose adjustments as currently advised by the algorithm, compared to the higher resolution of dose adjustments (1-s intervals) using existing technology, such as target controlled infusion (TCI) pumps. As the initiative for this study was taken by a USA based research center, the reason for not using TCI technology is probably related to the disturbing reality that nowadays no commercialized version of effect-site controlled TCI is approved by FDA in the US [6]. The reasons for the non-approval in the USA of a widespread and safe technology has a complex history and would lead me too far in this editorial [7-9]. But the worldwide acceptance of TCI technology does provide reassurance though, that effect-site controlled TCI pumps are easily applicable devices for targeting and maintaining predefined effect-site concentrations over time for many commonly used anesthetic drugs. The higher resolution of the dose adaptation of effect-site controlled TCI pumps 
result in the lowest prediction errors possible and therefore, it remains uncertain whether the new algorithm proposed by Tams et al. is a step forward towards optimal anesthetic drug titration? Prospective validation of performance and safety of the dose adaptations in 20-min intervals should be provided before a safe use can be advocated.

The innovative part of a TCI pump is that a clinician can titrate directly within the "effect-site concentration-domain" instead of in the "dose-domain" [9]. The administration of a single dose in a population of patients will result in a range of plasma-concentrations and drug effects due to interindividual differences in pharmacokinetic and -dynamic properties. The variability in effect is large and it must be advantageous to adjust the dose according to demographic covariates that significantly contribute to this biological variability. For propofol, remifentanil and most opioids such covariates have been identified and implemented in pharmacokinetic-dynamic models. Consequently, a direct titration towards the effect-site concentration using computercontrolled pumps will result in a lower degree of variability in the observed effect, due to the subtle adjustments of the administered dose over time compared to manual control.

It is important to understand that the effect-site concentration is not representing a predicted effect in the individual patient, but rather depicts the average effect of a population with comparable demographic characteristics. An example clarifies how this important difference should be interpreted and how crucial it is to strive for low prediction errors in the model performance: if a hypothetical effect-site model for propofol predicts that a steady-state effect-site concentration of $4 \mu \mathrm{g} / \mathrm{ml}$ represents a bispectral index (BIS) of 50, it does not mean that all patients titrated to that target will end up with a steady-state BIS of 50. Rather, the titration towards identical effect-site concentrations in many patients will result in a mean BIS of 50 in that population. This information is only useful for clinical application if the prediction error of the model is sufficiently small to evoke a clinically relevant performance. In a "good" predictive model, characterized by small prediction errors, most cases will reach a steadystate BIS in "close range" of the desired target. In our example, when most patients end up with a BIS between 40 and 60 , the prediction of the model can be considered reproducible and clinically useful, even though this example still represents a $20 \%$ prediction error! Despite of the error, only a minority of cases will end up with a deviating BIS compared to the expectations (higher than 60 or lower than 40). In these cases an adjustment of the initially chosen effect-site concentration $(4 \mu \mathrm{g} / \mathrm{ml}$ in our example) will be mandatory to maintain the BIS closer to 50 . The observation of such a case does not make the model "bad" or "useless". In our hypothetical example, a "bad" model would be characterized by high prediction errors $(>20 \%)$ that result in most patients with BIS values ranging between 20 and 80 , making the model much less attractive for clinical use. This example demonstrates the importance of using drug titration methods with high precision in order to guarantee the clinical performance.

The predictions of the response surface interaction model should also be interpreted as a population mean effect. For example, if a single patient is targeted towards two effect-site concentrations of respectively propofol and remifentanil, the response surface model will predict a corresponding probability of tolerance of laryngoscopy (PTOL). A PTOL of 90\% means that whenever multiple patients with similar demographic characteristics receive identical effect-site concentrations, only $90 \%$ of them will be tolerant for laryngoscopy and $10 \%$ will move in response. Consequently, even when a patient moves while the predicted PTOL is $90 \%$, it does not make the prediction a failure. Interaction models should rather be considered as diagnostic tools to identify the "more sensitive" versus "more resistant" patient compared to the average expected population effect. It provides confirmation that a patient's pharmacodynamic sensitivity for the drug is within or at the outer limits of what we commonly would expect compared to the "normal" response in a population of patients. This information allows adaptation of drug titration for the remainder of the procedure and eventually leads to improved outcome, such as reaching more predictable recovery conditions for each patient.

The clinical use of a population derived effect prediction requires some training for the user, but the examples above illustrate that this can lead to useful information for individual patients also. However, staying within the "dose domain" for optimizing titration adds a potential source of increasing prediction errors of all model derived estimations, posing a challenge for the proposed algorithm. Even though Tham et al. provide evidence of feasibility to improve recovery conditions, this evidence is depending on the assumption that the data input is done perfectly. The selection of primary endpoints and other methodological assumptions do not allow to use the acceptability study as a relevant prospective validation of performance. For example, the selection of the patient population (minors) is most cumbersome as the demographic characteristics of the pharmacokinetic-dynamic models for propofol, remifentanil and fentanyl are all derived from an adult population ( $>18$ years). The teenager population is currently the least well described population concerning the interaction characteristics of anesthetic drugs, and much of the information we do have, is derived from extrapolation of pediatric and adult populations [10]. Therefore, it will require a new prospective validation study, with an adequate selection of patient population and recovery parameters as primary endpoint, to demonstrate that this step back towards the "dose domain" may become an equally effective alternative for improving 
drug titration compared to titration into the "concentration domain" using TCI pumps.

\section{References}

1. Tams C, Syroid N, Vasilopoulos T, Johnson K. Optimizing intraoperative administration of propofol, remifentanil, and fentanyl through pharmacokinetic and pharmacodynamic simulations to increase the postoperative duration of analgesia. J Clin Comput Monit. 2019. https://doi.org/10.1007/s10877-019-00298-9.

2. Tams C, Johnson K, Seubert C. Acceptance of a propofol and remifentanil infusion dosing algorithm to optimize postoperative emergence and analgesia. J Clin Comput Monit. 2019. https://doi. org/10.1007/s10877-019-00295-y.

3. Lapierre C, Johnson K, Randall B, White J, Egan T. An exploration of remifentanil-propofol combinations that lead to a loss of response to esophageal instrumentation, a loss of responsiveness, and/or onset of intolerable ventilatory depression. Anesth Analg. 2011;113(3):490-9.

4. Kern S. Opioid hypnotic synergy. Anesthesiology. 2004;100:1373-81.
5. Bouillon T. Pharmacodynamic interaction between propofol and remifentanil regarding hypnosis, tolerance of laryngoscopy, bispectral index, and electroencephalographic approximate entropy. Anesthesiology. 2004;100:1353-72.

6. Egan TD, Shafer SL. Target-controlled infusions for intravenous anesthetics. Surfing USA Not! Anesthesiology. 2003;99:1039-41.

7. Absalom AR, Glen JI, Zwart GJ, Schnider TW, Struys MM. Target-controlled infusion: a mature technology. Anesth Analg. 2016;122(1):70-8.

8. Schnider TW, Minto CF, Struys MM, Absalom AR. The safety of target-controlled infusions. Anesth Analg. 2016;122(1):79-85.

9. Struys MM, De Smet T, Glen JI, Vereecke HE, Absalom AR, Schnider TW. The history of target-controlled infusion. Anesth Analg. 2016;122(1):56-69.

10. Eleveld DJ, Colin P, Absalom AR, Struys MMRF. Pharmacokinetic-pharmacodynamic model for propofol for broad application in anaesthesia and sedation. Br J Anaesth. 2018;120(5):942-59.

Publisher's Note Springer Nature remains neutral with regard to jurisdictional claims in published maps and institutional affiliations. 